every 5 cigarettes smoked daily, $95 \%$ confidence interval $1 \cdot 11$ to $2 \cdot 14$ ) but was not a risk factor for sudden infant death syndrome in infants over 24 weeks. On the other hand, when paternal smoking in the absence of maternal smoking was studied there was no significantly increased risk for the young infants with sudden infant death syndrome. The older infants who died were, however, at a significantly increased risk of sudden infant death syndrome if their fathers smoked (estimated relative risk $=2 \cdot 81$ for every $j$ cigarettes smoked daily, $95 \%$ confidence interval $1 \cdot 11$ to $7 \cdot 15$ ).

There are two types of explanation for how smoking could be causally related to postnatal deaths: smoking during pregnancy may leave the newborn infant more vulnerable, or postnatal passive smoking by the infant may increase the risk of death. These data provide some evidence for the passive smoking mechanism in older infants but not in younger infants, in whom maternal smoking during pregnancy appears to be an important factor lasting well beyond the neonatal period.

J P NICHOLL

ALICIA O'CATHAIN

Medical Care Research Unit,

Sheffield Medical School

Sheffield S10 2RX

1 Knowelden J, Keeling J, Nicholl JP. A multicentre study of post-neonatal mortlity. London: HMSO, 1985.

\section{Computer viruses}

Dr John Asbury points out that "a computer virus is a small piece of computer code which has been maliciously inserted on computer storage media" (23 July, p 246). Although this is true in most cases, there remains the possibility that some of these viruses have "evolved" out of random mutations of computer programs.

Some computer viruses are extremely small, consisting of short lengths of machine code. There are many small machine code lengths built in as part of the operating systems of computers to fulfil various tasks, such as reading, writing, and erasing data. Anyone who has ever used a computer will be extremely familiar with computer failures, or "crashes," when all the data in a computer's memory are scrambled and data are lost. This scrambling consists of random substitution of the computer memory locations with tiny fragments of machine code one or two bytes long. It is entirely possible, considering the number of times such events occur and the few substitutions required to mutate an existing length of machine code into a virus (analogous to base pair substitution in DNA), that such viruses will be thrown up quite regularly. Most will be fatal mutations and will wipe themselves out. Others will be stillborn, stable but nonfunctioning. Even fewer will emerge as infectious viruses. According to Darwinian natural selection, the viruses that are adapted to survive hidden inside computer systems will be the most successful and may proliferate, either as a result of the initial mutation or as a result of subsequent non-fatal mutations

Thus it is that life of a sort may have evolved in the computer systems designed by humans. The worrying thing is, as Dr Asbury suggests, that these new viruses may be very dangerous indeed, much more so than their biological counterparts. The AIDS virus may eventually kill many thousands of people. Yersinia pestis killed many millions in the Black Death. But a lively virus in the control system of an intercontinental ballistic missile may prove a greater human pathogen than any mere biological microbe.

JOHN CROALL

Public Health Laboratory,

William Harvey Hospital

Willesborough,

Ashford,

Kent TN24 OLZ

\section{Drug Points}

\section{Hypomagnesaemia and hypocalcaemia after treatment with mitozantrone}

Drs K D Griffrths and D H Parky (Y Ybyty Gwynedd, Bangor, Gwynedd LL57 2PW) write: Hypomagnesaemia hypocalcaemia, and hypoparathyroidism have been reported in patients with acute leukaemia and breast carcinoma after chemotherapy. ${ }^{12}$ We describe a patient with metastatic breast carcinoma who was given chemotherapy which included mitozantrone and developed hypomagnesaemia and hypocalcaemia apparently unrelated to hypoparathyroidism. As far as we are aware these biochemical abnormalities have not been reported with mitozantrone.

A 52 year old woman was referred with recurrent breast carcinoma eight years after a left mastectomy and bilateral salpingo-oophorectomy. After surgery she was treated with tamoxifen and aminoglutethimide and had been receiving thyroxine for several years. Nine months before this referral she had received radiotherapy to the sternum and ribs for painful metastases. Examination showed a mass in the contralateral breast, lymphadenopathy in the neck, and a mass in the left lower abdomen. Routine biochemical investigations gave normal results.

One month later, just before cytotoxic treatment was started, the results of biochemical investigations were: normal urea, creatinine, and albumin concentrations; potassium concentration normal at 3.6 $\mathrm{mmol} /$; calcium concentration $2.09 \mathrm{mmol} / \mathrm{l}$; alkaline phosphatase activity $484 \mathrm{U} / 1$; aspartate transaminase activity $149 \mathrm{U} / \mathrm{l}$; alanine transaminase activity $83 \mathrm{U} / \mathrm{l}$; and $\gamma$-glutamyltranspeptidase activity $33 \mathrm{U} / \mathrm{l}$. An isotope bone scan confirmed multiple areas of increased uptake consistent with widespread metastases.

Chemotherapy with mitozantrone $20 \mathrm{mg}(10 \mathrm{mg}$ $\left.\mathrm{m}^{2}\right)$, cyclophosphamide $1.2 \mathrm{~g}\left(0.6 \mathrm{~g} / \mathrm{m}^{2}\right)$, and vincristine $2 \mathrm{mg}$ was given intravenously. Four days later she was admitted to hospital as an emergency with a grand mal fit having had persistent nausea, vomiting, and diarrhoea. The abnormal biochemical finding were: potassium concentration $2.1 \mathrm{mmol} / 1$, chloride concentration $93 \mathrm{mmol} / \mathrm{l}$, aspartate transaminase activity $73 \mathrm{U} / 1$, alanine transaminase activity $30 \mathrm{U} / \mathrm{l}$ $\gamma$-glutamyltranspeptidase activity $25 \mathrm{U} / \mathrm{l}$, calcium concentration $1.54 \mathrm{mmol} / \mathrm{l}$ (with a normal albumin concentration), and phosphate concentration 0.91 $\mathrm{mmol} / \mathrm{l}$. Magnesium concentration measured a day later was $0.19 \mathrm{mmol} / \mathrm{l}$. Parathyroid concentration was $0.7 \mathrm{ng} / \mathrm{ml}$ (reference range less than $0.2 \mathrm{ng} / \mathrm{ml}$ ) and vitamin D concentration was normal. A random measurement of urinary calcium did not show a significantly increased excretion. No brain metastases were evident on computed tomography.

She was treated with intravenous and oral calcium supplements, vitamin D (as alfacalcidol), and intermittent intravenous magnesium sulphate infusions. The calcium concentration fell to $1 \cdot 20 \mathrm{mmol} / 1$ after admission in spite of replacement treatment, and magnesium concentrations fell rapidly when infusions were stopped, rarely rising above $0.5 \mathrm{mmol} / \mathrm{l}$. The serum albumin concentration was always within normal limits. Initially there was considerable clinica improvement, but continuous replacement treatmen with calcium and vitamin $\mathrm{D}$ was required to maintain normal serum calcium concentrations.

The initial response to the cytotoxic drugs was complete disappearance of soft tissue disease. Because of the metabolic complications, however, no further chemotherapy was given, and she died of progressive metastatic disease five months later

The grand mal fit was probably caused by rapid onset of hypocalcaemia and hypomagnesaemia. This rapid onset, occurring within four days, was much faster than has been reported with other drugs having this effect. The probable explanation is that the chemotherapy induced proximal renal tubular damage causing severe loss of magnesium with a concurrent reduction in serum calcium concentration. This would be supported by the low concentrations of potassium and phosphate. The vomiting and diarrhoea mav also have been contributory. Similar observation have been made with different cytotoxic drugs, ${ }^{3}$ but, unlike Freedman et al,' we found no evidence of hypoparathyroidism. The side effects of the cytotoxic drugs may have been enhanced by pre-existing impaired liver function related to hepatic metastases The doses were related to body surface area, and because the patient was grossly overweight, the dose of drug given mav also have been important.

We consider that these profound metabolic abnormalities were probably caused by the cytotoxic chemotherapy, perhaps by inducing renal tubular damage. The case was unusual in that although the patient received continuous calcium, vitamin $\mathrm{D}$, and magnesium replacement until the time of her death satisfactory control was never achieved. As far as we know this has not been reported previously in association with mitozantrone.

I Freedman DB, Shannon M, Dandona P, Prentice HG, Hoffbrand AV. Hvpoparathvroidism and hypocalcaemia during treatment for acute leukaemia. Br.Med 7 1982:284:700)-2.

Gome\%-Camphera FJ, Gonzales P, Camillo A, Estelles MC. Rengel .M. Cisplatin nephroroxicity: ssmptomatic hypomagnesaemia and renal failure. Intemutional foumul of Viphrolon 1986:7:151-2

W'omer RB, Pritchard J, Barratt TM. Renal toxicity of cisplatin in children. Y Pediatr 1985:106:659-63.

\section{Small bowel perforation associated with an excessive dose of slow release diclofenac sodium}

Mr M DEAKIN and others (Queen Elizabeth Hospital, Birmingham B15 2TH) write: Intestinal perforation associated with the ingestion of indomethacin is a well documented but uncommon side effect with a high risk of death. There have been seven deaths among the 18 cases reported to the Committee on Safety of Medicines (personal communication). Osmosin, long acting preparation of indomethacin, was withdrawn because of a number of reported perforations, possibly due to increased local toxicity associated with the formulation and drug delivery system.' The risk of perforation is also present with other non-steroida anti-inflammatory drugs thought to have a lower incidence of side effects.

A 70 year old man was referred with a 24 hour history of pain in the left iliac fossa. He had a fever anc signs of localised peritonism in his left iliac fossa. Twenty seven years previously he had had a laminectomy for a prolapsed intervertebral disc. He had suffered with continuous low back pain for many years and had been taking a combination of dipipanon hydrochloride and cyclizine hydrochloride (Diconal) twice daily for two years. 'Two weeks before admission he had been prescribed the recommended dose of diclofenac sodium, $100 \mathrm{mg}$ daily, in a sustained release preparation (Voltarol Retard). During the week before admission he had increased the dose to $100 \mathrm{mg}$ four times daily.

An initial diagnosis of acute diverticulitis was made and he was treated with parenteral antibiotics. His fever and abdominal tenderness resolved over 48 hours. Six days after admission he developed increasing abdominal distension and then had a sudden onset of generalised pain and tenderness. A laparotomy was performed, and he was found to have two perforation in the terminal ileum, each about $3 \mathrm{~mm}$ in diameter. The terminal ileum otherwise seemed to be normal. A biopsy specimen from the edge of a perforation showed no specific features. The perforations had sealed initially and had remained confined within small abscess cavity beside the sigmoid colon in the left iliac fossa, thus accounting for his localised signs at presentation. The abscess had ruptured subsequently into the peritoneal cavity. The two holes were oversewn with cat gut, and he made an uneventful recovery. Blood cultures and faecal samples were negative for salmonella.

To our knowledge this is the first reported case of small bowel perforation associated with the ingestion of slow release diclofenac sodium (a phenylalkanoic derivative). Non-steroidal anti-inflammatory drugs, including indomethacin and fenamic acid derivatives, given orally or parenterally to rats cause small bowel perforation within three or four days. ${ }^{3+}$ This effect is dose dependent, is believed to be caused by reduced prostaglandin synthesis by the small bowel mucosa, and can be prevented by giving prostaglandin $\mathrm{E}_{2}$

Our patient was suffering from pain that had been difficult to control over a long time, and he had ncreased his dose of diclofenac sodium to four times the recommended dose. The perforation could have been caused by high local drug concentrations, a factor that might have been exacerbated by taking the combination of dipipanone hydrochloride and 
cyclizine hydrochloride, which could have slowed his intestinal transit rate. Systemic toxicity is an alternative possibility. In view of the animal studies small bowel perforation is not an unexpected side effect, especially in association with the high dose of diclofenac sodium that this man was taking.

Non-steroidal anti-inflammatory drugs are often prescribed for pain that is unremitting and difficult to control, but their therapeutic window is small. With high dose slow release preparations it is easy to reach harmful concentrations by taking relatively few tablets. Patients taking slow release preparations of non-steroidal anti-inflammatory drugs should be warned specifically of the dangers of increasing the dose beyond that recommended. The risk of localised toxicity may be increased when non-steroidal antiinflammatory preparations are taken in conjunction with opiates or their derivatives, which decrease intestinal motility.

1 Day TK. Intestinal perforation associated with osmotic slow release indomethacin capsules. Br Med F 1983;287:1671-2.

2 Langman MJS, Morgan L, Worrall A. Use of anti-inflammatory drugs by patients admitted with small or large bowel perforaions and haemorrhage. BrMed f 1985;290:347-9.

3 Kent TH, Cardelli RM, Stander FW. Small intestinal ulcers and intestinal flora in rats given indomethacin. Am $\mathcal{J}$ Pathol 1969;54:237-45

4 Wax J, Cliger WA, Varner P, Bass P, Winder W. Relationship of the enterohepatic cycle to ulcerogenesis in the rat small bowel with flufenamic acid. Gastroenterology 1970;58:772-80.

5 Robert A. An intestinal disease produced experimentally by a prostaglandin deficiency. Gastroenterology 1975;69:1045-7.

\section{Unilateral deafness as a complication of the mumps, measles, and rubella vaccination}

Drs J Nabe-Nielsen and B Walter (Audiological Department, Aalborg Hospital, DK-9000 Aalborg, Denmark) write: A 7 year old girl, who had been found to have normal hearing by pure tone audiometry two years earlier, received a mumps, measles, and rubella vaccination without any immediate complications. Eleven days later she had a slight fever which lasted for two days. She then complained that she was deaf in her left ear. She had no dizziness, headaches, or earaches.

One month after the vaccination an ear, nose, and throat specialist found that she had a profound hearing impairment in her left ear by pure tone audiometry.

Ten weeks after the vaccination she had no complaints apart from the hearing loss. Pure tone audiometry showed anacusis in her left ear. In her right ear the hearing level was $0 \mathrm{~dB}$, with normal speech discrimination. No contralateral stapedius reflexes could be elicited from either ear. Ipsilateral stapedius reflexes were present in the right ear. Tympanometry was normal in both ears. Examination findings of the ears, nose, and throat were otherwise normal. Caloric testing showed no response in either ear to warm water and only weak but equal response to cold water. Postrotatory nystagmus was normal.

Eleven weeks after the mumps, measles, and rubella vaccination IgG antibodies to the mumps virus were found. Immunoglobulins $M$ and $A$ could not be found. The girl had never had symptoms of mumps. Her hearing impairment has not changed during the past 11 months, and she still has no other complaints.

Mumps is usually a benign disease, but it is an important cause of unilateral hearing impairment in children. With the development of the combined mumps, measles, and rubella vaccine vaccination should become more widespread, so reducing the amount of unilateral hearing loss in children. Adverse reactions to mumps, measles, and rubella vaccination are mainly in the form of fever. There are few serious complications, and most are reversible.

The Health Department in Denmark knows of no other case of hearing impairment after mumps, measles, and rubella vaccination. Only two cases of unilateral deafness have been reported before as a result of mumps vaccination. ${ }^{23}$

In our patient it seems reasonable to believe that the unilateral anacusis was caused by the mumps component of the mumps, measles, and rubella vaccine. This seems to be a rare complication, but it is important to be aware of this possibility during the first years of the vaccination programme, as it is only in older children that we will be able to discover this side effect. When nearly all children over 3 years of age have been vaccinated the discovery of unilateral deafness will be delayed and the relation to the vaccine will be difficult to prove.

Peltola $\mathrm{H}$, Hejnonen OP. Frequency of true adverse reactions to measles mumps rubella vaccine. Lancet 1986; ; $939-42$.

2 Hayden GF, Preblud SR, Orenstein WA, Conrad JL. Curren status of mumps and mumps vaccine in the United States. Pediatrics 1978;62:965-8.

3 Healy CE. Mumps vaccine and nerve deafness. Am 7 Dis Child 1972;123:612.

\section{Points}

\section{ABC of Eyes: Cataracts}

Mr D P Choyce (Westcliff on Sea, Essex SS0 8LR) writes: Messrs A R Elkington and P T Khaw clearly explained the current treatment of cataracts (25 June, p 1787). It is good to read that routine lens implantation is now accepted without question in most cases. They may, however, have missed a golden opportunity to extol the virtues of secondary lens implantation. There must be at least 100000 patients in the United Kingdom who had their cataracts removed before primary lens implantation became accepted practice not much more than five years ago whose visual rehabilitation was inhibited by reliance on cataract spectacles or contact lenses, the limitations of which were exposed by Messrs Elkington and Khaw. Most of these patients no longer attend hospital eye clinics (unless something has gone wrong with their aphakic eye(s)) and are generally unaware that they have not missed the boat, so to speak, and that they too could enjoy all the advantages of pseudophakic vision if only they asked. The arrival a few years ago of viscosurgery, of which the most widely used example is sodium hyaluronate, has rendered both primary and secondary lens implantation much safer. Perhaps this additional boon could have been briefly mentioned also.

Dr David GilberT (London SE21) writes: Messrs A R Elkington and P T Khaw (25 June, p 1787) stated that there is no effective medical treatment for established cataracts. This should be qualified with respect to central cataracts, in which, so long as the periphery of the lens remains clear, the use of a mydriatic agent can improve functional vision by increasing the ratio of focused to scattered light reaching the retina, particularly under bright ambient conditions. Ac commodation and adaptation are, of course, lost, but these losses can be compensated for by appropriate spectacles such as photochromic trifocals. My own central cataracts have been managed in this way for over 10 years. It is a treatment option that is worth keeping in mind for the occasional patient who could benefit from it.

\section{Organs for transplantation}

Dr GREY E B Giddins (Royal National Orthopaedic Hospitals, Stanmore, Middlesex) writes: In response to Professor Geoffrey D Chisholm's article on organ transplantation (21 May, p 1419) several correspondents have pointed out the inaccuracy of his statement that "a patient not on a respirator can never be a donor" and have correctly stated that kidneys and corneas can be taken from patients who are not on respirators (2 July, p 68; 16 July, p 201). Sadly, once again, as on donor cards, there has been no mention of bones. Bone transplantation, which was first reported in $1908,{ }^{1}$ remains a neglected art in Britain especially when compared with that in North America, where most major orthopaedic centres have extensive transplant programmes. Bones can be harvested for fresh immediate use or delayed use almost whenever other organs are taken. Moreover, bone transplantation does not require cross matching, immunosuppres sion, or a "beating heart" donor, and only for fresh grafts is there a need for immediately available recipients. The uncommonness of bone harvesting in Britain represents a considerable waste of resources The inclusion of bones on donor cards may heighten both public and medical awareness and help to make better use of this facility.

1 Lexer E. Joint transplantation. Clinical Orthop 1985;197 4-10.

\section{Myocardial microthrombi in systemic} lupus erythematosus

Professor Priscilla Kincaid-Smith (Department of Medicine, Royal Melbourne Hospital, Victoria 3050 Australia) writes: The case reported by Dr J H Brown and others (28 May, p 1505) is similar to a series of 12 women with pregnancy related acute thrombotic microangiopathy and impaired renal function which we reported recently (in press). The common factor in these young women was the presence of a circulating lupus anticoagulant. Eight of the 10 women in whom the test was performed had a positive test for antiphospholipid (anticardiolipin) antibodies. These patients had fibrin thrombi in arteries, arterioles, and glomerular capillaries as seen in biopsy specimens. These findings are typical of thrombotic microangiopathy. Only two of 23 pregnancies in these women were successful, and pregnancy precipitated the acute thrombotic microangiopathy in most of our patients. One, however, had had a previous episode of throm botic microangiopathy related to taking an ora contraceptive agent. This prompts the question whether the 22 year old patient described by the authors was using oral contraceptives before her fulminating fatal microangiopathy. [The authors confirm that the patient had never taken oral contraceptives and was not pregnant-ED.]

\section{Which patients should undergo laparoscopy?}

Dr Hamish McA Foster (Hunter Oncology Centre, Waratah, New South Wales 2298, Australia) writes: agree with $\mathrm{Mr} S$ Patterson-Brown and others that a laparoscopy should be performed on all women who present with a clinical diagnosis of acute appendicitis (14 May, p 1363). The clinical diagnosis of acute appendicitis in women is difficult, especially in those of childbearing age. Accuracy rates can be as low as about a half. ${ }^{1}$ In the North Solomons province of Papua New Guinea laparoscopy has been used before operation in virtually all women with suspected appendicitis in an attempt to reduce the rate of unnecessary exploration and appendicectomy. Over three years 39 women with suspected appendicitis had a laparoscopy. Twenty nine required no further operation and were observed or received antibiotics for pelvic inflammatory disease. Eight had appendicectomies, and two required a laparotomy for pelvic disease. Unnecessary appendicectomy was thus avoided in 29 of the 39 patients ( $74 \%$ ), an important saving in a country where surgical resources are scarce. When the appendix could not be seen at laparoscopy an appendicectomy was carried out. When the appendix was normal and no other cause of the patient's abdominal pain could be found the patient was either observed in hospital or had an immediate appendicectomy. We have not found that laparoscopy is useful in men with suspected appendicitis in the North Solomons as most of them present with signs of late appendicitis (a mass or peritonitis). Laparoscopy is unnecessary and hazardous in such patients.

1 Chang FC, Hogle HH, Welling DR. The fate of the negative appendix. Am F Surg 1973;126:752-4.

\section{Undergraduate medical education}

Professor R M HARDEN (Centre for Medical Education, University of Dundee, Dundee DDl 9SY writes: Dr Brian Jolly's letter about medical education in Britain (9 July, p 136) is misleading. His estimate of five educationalists working full time on medical educational problems in British medical schools is low. In this unit alone we have eight staff working full time in this subject. Dr Jolly's information about the lack of training programmes in medical education is equally out of date. In Dundee, in addition to three week courses in medical education, which have so far attracted more than 600 medical teachers from the United Kingdom and overseas, we now offer a distance learning diploma course in medical education. This was designed to meet the needs identified by Professor Peter Richards for "clinical teachers who understand the complexity of medicine to think about patterns of medical education for the future" (7 May, p 1279). The first two doctors have now completed the course successfully and 50 are enrolled currently. 